\title{
Electrophysiology of Object Naming in Primary Progressive Aphasia
}

\author{
Robert S. Hurley, ${ }^{1}$ Ken A. Paller, ${ }^{1}$ Christina A. Wieneke, ${ }^{1}$ Sandra Weintraub, ${ }^{1}$ Cynthia K. Thompson, ${ }^{1}$ \\ Kara D. Federmeier, ${ }^{2}$ and M.-Marsel Mesulam ${ }^{1}$ \\ ${ }^{1}$ Cognitive Neurology \& Alzheimer's Disease Center, Northwestern University, Chicago, Illinois 60660, and 2Beckman Institute, University of Illinois, \\ Urbana, Illinois 61801
}

\begin{abstract}
Primary progressive aphasia (PPA), a selective neurodegeneration of the language network, frequently causes object naming impairments. We examined the $\mathrm{N} 400$ event-related potential (ERP) to explore interactions between object recognition and word processing in 20 PPA patients and 15 controls. Participants viewed photographs of objects, each followed by a word that was either a match to the object, a semantically related mismatch, or an unrelated mismatch. Patients judged whether word-object pairs matched with high accuracy (94\% PPA group; 98\% control group), but they failed to exhibit the normal N400 category effect (N400c), defined as a larger N400 to unrelated versus related mismatch words. In contrast, the N400 mismatch effect $(\mathrm{N} 400 \mathrm{~m})$, defined as a larger N400 to mismatch than match words, was observed in both groups. N400m magnitude was positively correlated with neuropsychological measures of word comprehension but not fluency or grammatical competence, and therefore reflected the semantic component of naming. After ERP testing, patients were asked to name the same set of objects aloud. Trials with objects that could not be named were found to lack an $\mathrm{N} 400 \mathrm{~m}$, although the name had been correctly recognized at the matching stage. Even accurate overt naming did not necessarily imply normal semantic processing, as shown by the absent $\mathrm{N} 400 \mathrm{c}$. The $\mathrm{N} 400 \mathrm{~m}$ was preserved in one patient with postsemantic anomia, who could write the names of objects she could not verbalize. N400 analyses can thus help dissect the multiple cognitive mechanisms that contribute to object naming failures in PPA.
\end{abstract}

\section{Introduction}

The process by which we name objects seems largely automatic and effortless for most adults. Object naming deficits or "anomias," however, are a common symptom in aphasic patients with lesions in different nodes of the left perisylvian language network, including inferior frontal and temporal regions (Damasio et al., 2004). DeLeon et al. (2007) found that anomia in stroke aphasics is sometimes accompanied by errors during object-word matching tasks, suggesting that anomia in these patients may result from loss of knowledge of object names. This type of "semantic" anomia was most closely associated with damage to the left posterior temporal lobe. In contrast, some patients demonstrated output-specific naming impairments, such that they could write the names of objects but could not name them aloud. This type of "postsemantic" anomia was most closely associated with left inferior frontal damage.

Anomia is also common in patients with a neurodegenerative language disorder known as primary progressive aphasia (PPA)

Received June 17, 2009; revised Aug. 18, 2009; accepted 0ct. 22, 2009.

This work was funded by the National Institute on Deafness and Other Communication Disorders (DC008552) and the National Institute on Aging [AG13854 (Alzheimer's Disease Center)]. Additional support for R.S.H. was provided by the National Institute of Neurological Disorders and Stroke Training Program in the Neuroscience of Human Cognition (NS047987).

Correspondence should be addressed to Robert S. Hurley, Cognitive Neurology \& Alzheimer's Disease Center, Northwestern University, 320 East Superior Street, Searle 11-447, Chicago, IL 60660. E-mail: hurley@ u.northwestern.edu.

D0I:10.1523/JNEUROSCI.2912-09.2009

Copyright $(\odot 2009$ Society for Neuroscience $\quad 0270-6474 / 09 / 2915762-08 \$ 15.00 / 0$
(Mesulam, 1982). A diagnosis of PPA is made when patients exhibit anomia, agrammatism, or word comprehension deficits, when these language problems become worse over time, and when language impairments are unaccompanied by consequential cognitive decline in other domains during the initial stages of the disease (Mesulam, 2003). As with acquired aphasias, anomia in PPA can result from either semantic or postsemantic factors. Grossman and colleagues used voxel-based morphometry to correlate patterns of atrophy with confrontation naming scores in PPA patients (Grossman et al., 2004; McMillan et al., 2004). Whereas naming deficits in nonfluent patients, whose anomia is presumably mostly postsemantic, were correlated with atrophy in frontal areas, deficits in fluent patients were associated with atrophy in temporal areas.

Accurate naming requires knowledge of the object, knowledge of the word that denotes the object, linkage of the object representation to its corresponding lexical representation, and the capacity to retrieve and phonologically encode the appropriate word (Levelt et al., 1999; DeLeon et al., 2007; Mesulam et al., 2009). In the current study, we used electrophysiology to explore interactions between object and word representations in PPA. To our knowledge, this is the first group study of PPA using eventrelated potentials (ERPs). Our analyses focus on the N400, a negative-going potential that is sensitive to semantic incongruity and maximal $\sim 400 \mathrm{~ms}$ following the target stimulus (Kutas and Hillyard, 1980). The N400 is sensitive to semantic dysfunction in disorders such as Alzheimer's disease (Ford et al., 2001; Auchterlonie et al., 2002) and schizophrenia (Mathalon et al., 2002; Kiang et al., 
Table 1. Demographic and neuropsychological scores for PPA patients and controls

\begin{tabular}{|c|c|c|c|c|c|c|c|c|c|c|c|c|c|c|}
\hline ID & Gender & Age & $\begin{array}{l}\text { WAB AQ } \\
(\max 100)\end{array}$ & $\begin{array}{l}\text { BNT } \\
(\max 60)\end{array}$ & $\begin{array}{l}\text { Post-ERP } \\
\text { naming } \\
(\max 80)\end{array}$ & $\begin{array}{l}\text { PPT } \\
\text { Pictures } \\
\text { (max 52) }\end{array}$ & $\begin{array}{l}\text { PPT } \\
\text { Verbal } \\
(\max 52)\end{array}$ & $\begin{array}{l}\text { PPVT } \\
(\max 36)\end{array}$ & $\begin{array}{l}\text { WAB } \\
\text { Word Rec } \\
(\max 60)\end{array}$ & $\begin{array}{l}\text { WAB } \\
\text { SS } \\
(\max 20)\end{array}$ & $\begin{array}{l}\text { NAVS } \\
\text { Production } \\
(\max 20)\end{array}$ & $\begin{array}{l}\text { NAVS } \\
\text { Comp } \\
(\max 15)\end{array}$ & WPM & MLU \\
\hline P1 & $M$ & 69 & 92 & 59 & 79 & 51 & 51 & 35 & 60 & 18 & 15 & 13 & 104 & 9.5 \\
\hline $\mathrm{P} 2$ & $M$ & 60 & 80 & 59 & 78 & 52 & NT & 35 & 60 & 15 & 13 & 13 & 63 & 6.5 \\
\hline P3 & M & 66 & 86 & 56 & 73 & 52 & NT & NT & 60 & 17 & 12 & 15 & 101 & 7.9 \\
\hline P4 & $M$ & 63 & 97 & 58 & 80 & 50 & 52 & 36 & 60 & 20 & 15 & 15 & 141 & 7.9 \\
\hline P5 & $\mathrm{F}$ & 64 & 93 & 54 & 79 & 50 & NT & NT & 60 & 19 & 14 & 14 & 98 & 7.8 \\
\hline P6 & $M$ & 61 & 81 & 54 & 76 & 50 & NT & NT & 56 & 16 & 7 & 11 & 63 & 8.6 \\
\hline P7 & $M$ & 62 & 82 & 59 & 76 & 52 & 52 & 36 & 60 & 14 & 1 & 9 & 47 & 2.9 \\
\hline P8 & $M$ & 59 & 80 & 49 & 78 & 49 & 47 & 34 & 57 & 14 & NT & 10 & 55 & 5.0 \\
\hline P9 & $M$ & 58 & 87 & 54 & 79 & 52 & 52 & 34 & 60 & 15 & 13 & 11 & 119 & 10.5 \\
\hline P10 & $\mathrm{F}$ & 52 & 79 & 46 & 78 & 51 & 51 & 34 & 59 & 14 & 4 & 10 & 110 & 7.6 \\
\hline P11 & $\mathrm{F}$ & 75 & 97 & 53 & 75 & 49 & 49 & 35 & 60 & 20 & 13 & 15 & 158 & 13.9 \\
\hline P12 & $\mathrm{F}$ & 56 & 76 & 3 & 18 & 42 & NT & 14 & 46 & 17 & 15 & 15 & 167 & 9.6 \\
\hline P13 & $\mathrm{F}$ & 60 & 53 & 6 & 20 & 43 & 32 & 16 & 54 & 9 & 0 & NT & 82 & 7.3 \\
\hline P14 & $\mathrm{F}$ & 64 & 74 & 5 & 9 & 41 & NT & 31 & 57 & 16 & 9 & 13 & 127 & 7.4 \\
\hline P15 & $\mathrm{F}$ & 66 & 50 & 12 & 55 & 35 & 36 & 27 & 56 & 11 & NT & NT & 42 & 3.1 \\
\hline P16 & $M$ & 61 & 88 & 30 & 65 & 46 & NT & 18 & 59 & 18 & 10 & 13 & 83 & 10.1 \\
\hline P17 & $M$ & 59 & 91 & 52 & 69 & 52 & 51 & 34 & 60 & 19 & 6 & 12 & 86 & 6.3 \\
\hline P18 & $M$ & 66 & 90 & 21 & 62 & 48 & 48 & 34 & 56 & 19 & 15 & 15 & 83 & 7.6 \\
\hline P19 & $\mathrm{M}$ & 63 & 88 & 14 & 56 & 42 & 43 & 17 & 59 & 19 & 15 & 14 & 164 & 13.7 \\
\hline P20 & $\mathrm{F}$ & 56 & $42 / 65$ & $16 / 49$ & 1 & 51 & 50 & 30 & 60 & 1 & NT & 7 & NT & NT \\
\hline
\end{tabular}

Mean \pm SD

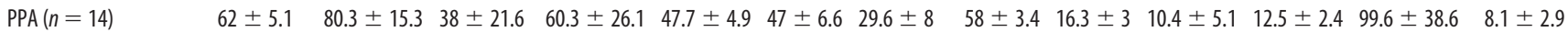

$$
\begin{aligned}
& \text { Controls }(n=14) \quad 62.7 \pm 6.2 \quad 99.6 \pm 0.7 \quad 58.6 \pm 1.5 \quad \text { NT } \quad 51.1 \pm 0.8 \quad 51.4 \pm 1 \quad 35.4 \pm 0.8 \quad 60 \pm 0 \quad 20 \pm 0 \quad 15 \pm 0 \quad 15 \pm 0 \quad 131.9 \pm 19.6 \quad 11.1 \pm 2.1
\end{aligned}
$$

AQ, Aphasia Quotient subtest; Word Rec, Auditory Word Recognition subtest; SS, Spontaneous Speech subtest; Production, noncanonical Sentence Production Priming subtest; Comp, Sentence Comprehension subtest; WPM, words per minute; MLU, mean length of utterance; max, maximum score; Post-ERP naming, confrontation naming test done after ERP testing; NT: not tested (some participants were unable to complete certain tests due to their level of impairment, or because tests were not yet added to the testing battery at the time of assessment).

2008). Hagoort et al. (1996) found that N400 potentials were of similar magnitude in controls and in aphasic patients with relatively intact word comprehension, but reduced in aphasic patients with comprehension deficits. In the current study, we recorded N400 potentials to words preceded by objects, simulating conditions involved in object naming, to explore the mechanisms of anomia in PPA.

\section{Materials and Methods}

Participants. Twenty PPA patients [ 12 male (M), 8 female (F)] and fifteen unimpaired controls (7 M, $8 \mathrm{~F}$ ) participated in this study. Diagnosis of PPA (Mesulam, 2003) required a history of language impairments in word finding, object naming, syntax, or word comprehension unaccompanied by consequential decline in other cognitive domains for at least the first 2 years of the disease. To meet research criteria for the presence of aphasia, patients were required to have abnormal performance on the Western Aphasia Battery (WAB) (Kertesz, 1982). Three patients were left handed. Controls were recruited from the Chicago area as well as from the Northwestern Alzheimer's Disease Center Clinical Core, and inclusion criteria consisted of English as a first language, right handedness, and no history of serious medical or psychiatric illness. Groups were equivalent in age (PPA mean: 62 years, control mean: 63 years; $t_{(33)}=$ $0.35, p=0.73$ ) and years of education (PPA mean: 16.6 years, control mean: 16.0 years; $\left.t_{(33)}=0.63, p=0.53\right)$.

Neuropsychological testing. All of the PPA patients and all but one control completed a battery of neuropsychological language tests within 1 week of ERP testing. Key test scores relevant to this study are shown in Table 1. Confrontation naming was assessed by the Boston Naming Test (BNT) (Kaplan et al., 1983), where participants are shown line drawings of concrete objects and are asked to name each one aloud. Semantic judgment was assessed by the Pyramids and Palm Trees Test (PPT) (Howard and Patterson, 1992), where participants are shown three items and asked to pick which one of two choices is most similar to the third, target item. Two conditions of this test were administered, one using pictures and one using object words. Single-word comprehension was assessed by the auditory word recognition subtest of the WAB and by a 36-item subset of the Peabody Picture Vocabulary Test (PPVT, moder- ately difficult items \#157-192) (Dunn and Dunn, 2006), where participants are given an array of pictures and asked to select an item after hearing its name aloud. Spontaneous grammatical production and grammatical comprehension were tested with two subtests of the Northwestern Assessment of Verbs and Sentences (NAVS) (C. K. Thompson, experimental edition). In the Sentence Comprehension subtest, patients are shown similar semantically reversible pairs of pictures denoting actions, and are given a sample noncanonical sentence aloud (e.g., "Pete saw the girl who was kissing the boy"), and are then asked to point to the corresponding picture. In the Sentence Production Priming subtest of the NAVS, patients are shown a picture depicting an action and are given a noncanonical sample sentence, and are then required to generate a similar sentence for another picture. Spontaneous speech samples were recorded while patients told the story of Cinderella from a wordless picture book. Fluency of speech was quantified based on these samples, including mean length of utterance and mean words per minute.

Object-word matching task. Pictorial stimuli for the ERP object-word matching task were 80 grayscale photographs, including 10 items from each of eight categories: domestic animals, foreign animals, body parts, fruits/vegetables, tools, clothing, kitchenware, and office supplies. All photographs were tested for naming reliability before the study, and $\geq 28 / 30$ younger adults provided the target name for each item. Matching pairs consisted of objects and written words referring to the same item. Related pairs were composed of an object and a nonmatching word from the same category (e.g., picture of a dog and the word "cat"). Unrelated pairs consisted of an item from a living category (e.g., an animal, cat) and an item from a manmade category (e.g., a tool, saw). Stimuli were counterbalanced such that each of the 80 items was presented three times as an object cue (in matching, related, and unrelated conditions), and also three times as a word target (once in each condition). Eighty filler trials (not analyzed), all matching pairs from the same living and manmade categories, were included to counteract a potential response bias for mismatched pairs, resulting in a total of 320 trials, equally divided between matching and nonmatching pairs.

Each object prime was followed by a word probe at a stimulus onset asynchrony of either 1 or $2 \mathrm{~s}$, split evenly in each condition. The word probe remained on the screen for $800 \mathrm{~ms}$, followed by a jittered intertrial 
interval of 3-5 s. Participants were instructed to press one mouse button if the two stimuli matched, and the other button for mismatches (related and unrelated). They were told their reaction times were being recorded, so they were to respond as quickly as possible while still pressing the correct buttons. They were also told to keep blinking to a minimum to prevent interference in the EEG recording. The ERP paradigm took $\sim 45$ min to complete, including time for breaks that were provided after every 80 trials.

EEG was recorded from 59 scalp electrodes in an elastic cap, after impedance was lowered to $5 \mathrm{k} \Omega$ or less. EEG signals were acquired using a bandpass of $0.05-200 \mathrm{~Hz}$, a sampling rate of $1000 \mathrm{~Hz}$, and a left mastoid reference (changed offline to averaged mastoids). Electro-ocular artifacts were monitored using electrodes placed below and lateral to the eyes. Data were epoched from -100 to $800 \mathrm{~ms}$ relative to word onset, and baseline corrected to the $100 \mathrm{~ms}$ prestimulus interval. Epochs with electro-ocular artifacts were excluded from analysis except when this resulted in loss of $>40$ of the 240 trials for any participant. In these cases (15 PPA patients and 7 controls), an eyeblink-correction algorithm was implemented. This algorithm was trained via principal components analysis to recognize each participant's blink signature, and this factor was then partialled out of EEG traces. Trials with eye movements were not affected by the blink-correction algorithm, and were excluded from analysis. In addition, trials with inaccurate match/nonmatch responses were also excluded. The mean number of trials remaining for ERP analyses (out of 240) was 203 in patients and 215 in controls.

Confrontation naming task. After ERP testing, PPA patients were shown the same set of 80 pictures used in the picture-word matching task. Each item appeared on the screen until the patient made a vocal response or indicated they would not be able to name the picture. Naming responses were recorded by the experimenter.

\section{Results}

\section{Group analyses: PPA versus control}

In a preliminary analysis, no interactions were found between stimulus-onset asynchrony interval ( 1 vs $2 \mathrm{~s}$ ) and match condition (match/related/unrelated) for accuracy, reaction times, or ERPs. Analyses reported below were thus conducted after collapsing trials across the two intervals.

Across conditions, PPA patients showed 94\% accuracy, demonstrating good comprehension of task instructions and ability to perform the object-word task. Controls performed the task with even higher accuracy $\left(98 \%, t_{(33)}=3.2, p=\right.$ $0.004)$. Whereas accuracy for related and unrelated mismatches was similar in controls $\left(t_{(14)}=1.5, p=0.15\right)$, PPA patients showed lower accuracy on related trials $\left(t_{(19)}=3.2, p=0.004\right)$ (Fig. 1a). Reactiontime and ERP analyses excluded trials with incorrect responses. Mean reaction times were slower in the PPA group than in the control group $\left(F_{(1,33)}=6.5, p=0.015\right)$ (Fig. $\left.1 b\right)$. A contrast to discern the mismatch effect (weights $=-2: 1: 1$ for match:related:unrelated) showed that reaction times were significantly slower for mismatched versus matching words in patients $\left(F_{(1,19)}=35, p<0.001\right)$ and in controls $\left(F_{(1,14)}=42, p<0.001\right)$. Responses were also significantly slower to related than to unrelated mismatches in both groups (patients, $t_{(19)}=2.9, p=0.009$; controls, $t_{(14)}=2.8, p=$ 0.01 ). Between-group differences were nonsignificant for both the mismatch effect $\left(t_{(32.5)}=0.82, p=0.42\right)$ and the related/unrelated $\operatorname{effect}\left(t_{(24.4)}=1.7, p=0.10\right)$.

For ERP analyses, mean amplitudes from 350 to $550 \mathrm{~ms}$ after word onset were used to quantify N400 potentials. Amplitude measurements from 15 bilateral central/posterior electrode locations were submitted to repeated-measures ANOVAs with

\section{a Accuracy (proportion correct)}

\begin{tabular}{|c|c|c|c|}
\hline & Match & Related & Unrelated \\
\hline Control & .97 & .99 & .99 \\
\hline PPA & .93 & .91 & .97 \\
\hline
\end{tabular}

\section{b Reaction Times (ms)}

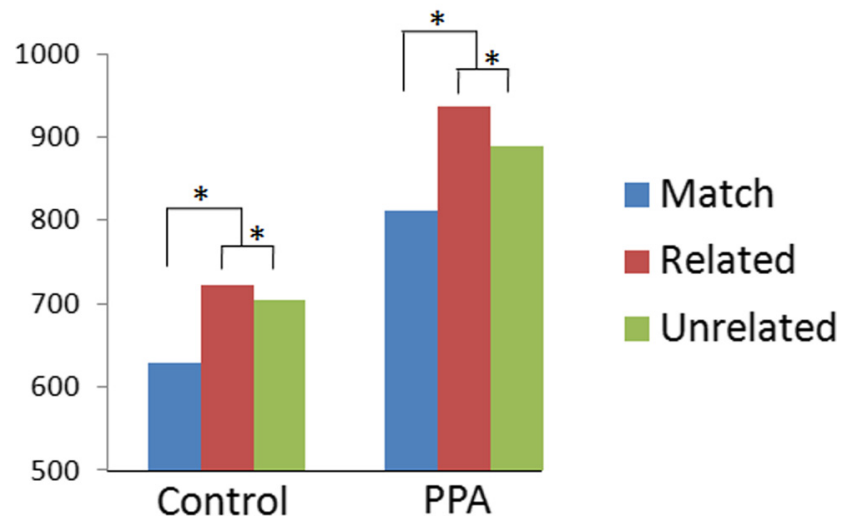

Figure 1. Behavior in the object-word matching task. Accuracy $(\boldsymbol{a})$ and reaction times $(\boldsymbol{b})$ for the control group $(n=15)$ and the PPA group $(n=20) .{ }^{*} p<0.01$.

\section{a Controls $(n=15)$}

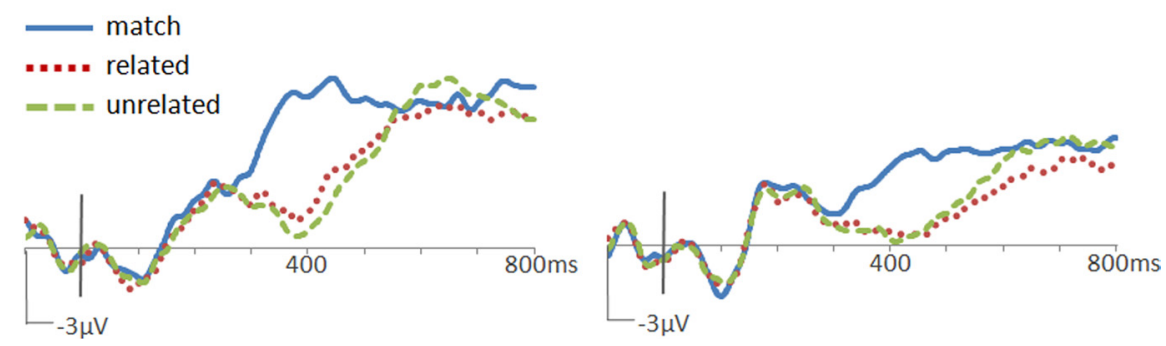

Figure 2. ERPs to words in the object-word matching task. Waveforms from the Czlocation are shown for the control group (a) and the PPA group $(\boldsymbol{b})$. Positive potentials are plotted up. $\mathrm{N} 400 \mathrm{~m}$ was evident in both groups as greater negative-going potentials trace). N400 c was evident in the control group as greater negative-going potentials to unrelated mismatches (dashed green trace) than to related mismatches (dotted red trace). electrode location as a factor. At these locations (shown in supplemental Fig. 1, available at www.jneurosci.org as supplemental material), differences between conditions were obvious in both PPA and control groups. Both PPA patients and controls showed more negative responses to mismatched (both related and unrelated) than to matching words, constituting an N400 mismatch effect (N400m). This effect was maximal at the central midline $(\mathrm{Cz})$ location (PPA: $2.4 \mu \mathrm{V}$; control: $3.3 \mu \mathrm{V}$ ). ERPs formed for each group are shown in Figure 2. As with the reaction time contrasts, responses on matching trials were compared with both related and unrelated trials via contrast weights of $-2: 1: 1$ in a repeatedmeasures ANOVA model. These contrasts revealed significant $\mathrm{N} 400 \mathrm{~m}$ effects in both the PPA $\left(F_{(1,19)}=30.8, p<0.001\right)$ and control $\left(F_{(1,14)}=36.5, p<0.001\right)$ groups. Results from a mixed-model ANOVA indicated that $\mathrm{N} 400 \mathrm{~m}$ did not differ in magnitude between groups $\left(F_{(1,33)}=1.3, p=0.26\right)$.

The timing of this mismatch effect was compared across groups by examining ERP amplitudes over consecutive $100 \mathrm{~ms}$ intervals after word onset (using the same bilateral central/ 
a

$\mathrm{N} 400 \mathrm{~m}$

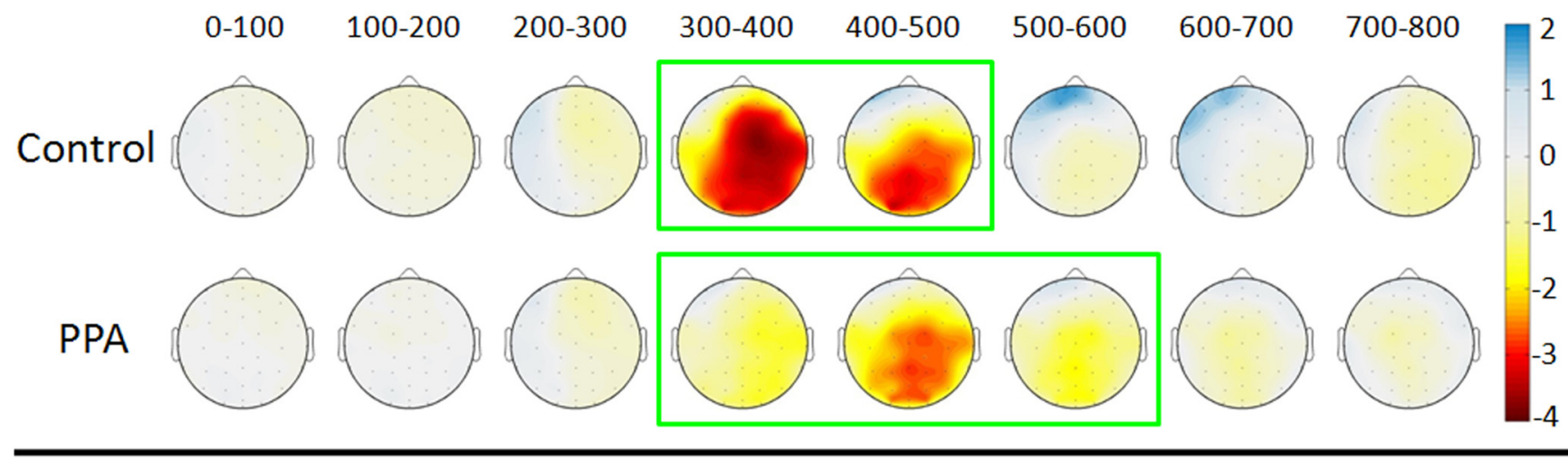

b

N400c

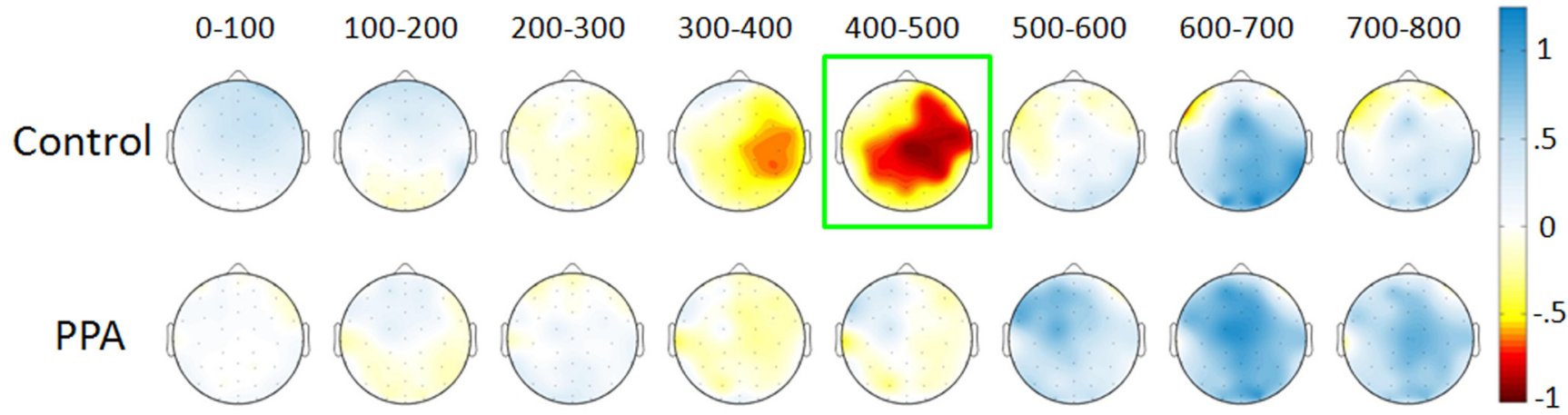

Figure 3. Topographic plots of $\mathrm{N} 400$ effects over $100 \mathrm{~ms}$ intervals. Negative potentials are shown in yellow/red and positive potentials are shown in blue, corresponding to microvolt values shown on the color scale, as interpolated across a schematic view of the head as viewed from above. $\boldsymbol{a}, \mathrm{N} 400 \mathrm{~m}$ amplitudes were calculated as mean amplitudes for the related mismatch condition minus the matching condition. Intervals are demarcated in green for significant N400 m effects for the bilateral central/posterior cluster. $\boldsymbol{b}, \mathrm{N} 400 \mathrm{c}$ amplitudes were calculated as mean amplitudes for the unrelated mismatch condition minus the related mismatch condition. Intervals are demarcated in green for significant N400c effects in the same electrode cluster.

posterior cluster). In controls, $\mathrm{N} 400 \mathrm{~m}$ was significant for intervals from 300 to $400 \mathrm{~ms}\left(F_{(1,14)}=28, p<0.001\right)$ and 400 to 500 $\mathrm{ms}\left(F_{(1,14)}=30.6, p<0.001\right)$. In PPA patients, $\mathrm{N} 400 \mathrm{~m}$ was significant for intervals from 300 to $400 \mathrm{~ms}\left(F_{(1,19)}=5.8, p=\right.$ $0.026), 400$ to $500 \mathrm{~ms}\left(F_{(1,19)}=39.6, p<0.001\right)$, and 500 to 600 $\mathrm{ms}\left(F_{(1,19)}=10.5, p=0.004\right)$, demonstrating a prolongation of the effect in this group. Topographic plots of N400m amplitude (represented by related minus matching values) in $100 \mathrm{~ms}$ intervals are shown in Figure $3 a$.

An N400 category effect (N400c), a more negative response to unrelated than to related mismatches, was evident in the control group from $\sim 350$ to $550 \mathrm{~ms}$. As topographic plots of N400c amplitude show in Figure $3 b$, N400c was slightly larger on the right than on the left side of the scalp in controls (measuring $0.63 \mu \mathrm{V}$ at electrode location C4). In contrast, N400c was not apparent in the PPA group (measuring $0.06 \mu \mathrm{V}$ at $\mathrm{C} 4$ ). For the bilateral central/posterior cluster, N400c was significant in the control group $\left(F_{(1,14)}=4.72, p=\right.$ $0.047)$ but not in the PPA group $\left(F_{(1,19)}=0.011, p=0.917\right)$. An analysis over $100 \mathrm{~ms}$ intervals showed that N400c in controls approached significance at $300-400 \mathrm{~ms}\left(F_{(1,14)}=3.39, p=0.087\right)$ and was reliable at $400-500 \mathrm{~ms}\left(F_{(1,14)}=4.93, p=0.043\right)$. N400c was not significant during any interval in the PPA group.

Absence of an N400c in PPA could reflect a legitimate processing difference distinguishing PPA patients from controls, or it could merely reflect increased variability or low signal:noise among the patients. To evaluate this possibility, N400c amplitudes were calculated in each individual by subtracting unrelated from related values $(350-550 \mathrm{~ms}$ at C4). N400c effects were robust in two-thirds of controls, whereas the distribution in patients was centered at zero (supplemental Fig. 2, available at www. jneurosci.org as supplemental material), consistent with the idea that $\mathrm{N} 400 \mathrm{c}$ was truly absent in patients.

The influence of the artifact correction that was applied as needed was examined via between-group comparisons between ERPs that did not require correction (eight controls) versus corrected ERPs (seven controls). A mixed-model repeated-measures ANOVA showed a nonsignificant group-by-condition interaction $\left(F_{(2,26)}=0.928, p=0.41\right)$, suggesting that $\mathrm{N} 400$ effects were not systematically influenced by whether or not electro-ocular artifact correction algorithms were applied.

Relationship between $\mathrm{N} 400 \mathrm{~m}$ and neuropsychological results Correlations between N400m amplitude and scores on neuropsychological tests of semantic and language functioning are shown in Table 2. Amplitudes were positively correlated with confrontation naming scores on the BNT, providing face validity that ERPs from the object-word matching task reflect the ability to name objects. Also, N400m amplitudes were strongly correlated with scores for single-word comprehension (WAB Auditory Word Recognition, Peabody Picture Vocabulary Test). Correlations were also found with the PPT, which involves making semantic decisions about objects and words. In contrast, N400m amplitudes were not correlated with postsemantic language measures, including fluency in spontaneous speech samples and 
grammatical production and comprehension. No correlations were found with $\mathrm{N} 400 \mathrm{c}$ values.

\section{Responses to named and unnamed items}

During the confrontation-naming phase after ERP testing, PPA patients were unable to correctly name many of the 80 objects previously seen during ERP testing (group mean = $25 \%$ naming errors). After artifact rejection, 8 of the 20 PPA patients had enough trials in each of the experimental conditions (match/related/unrelated) to generate averaged ERPs to named and unnamed items separately. These also happened to be the patients who made the most confrontation naming errors, failing to name between 14 and $89 \%$ of the 80 ERP stimuli (Table 1). This severely anomic subgroup (patients P12-P19) also failed to name $70 \%$ of BNT items. Naming scores for the experimental ERP objects were highly correlated with BNT naming scores $(r=$ $0.78, p=0.02)$. There were, however, rare discrepancies between the BNT and post-ERP confrontation naming scores. For example, P17, who was placed in the severly anomic group on the basis of post-ERP naming, had a BNT score in the range of the mildly anomic group. The majority of errors in the severely anomic subgroup consisted of "don't know" responses (73\%), followed by semantic paraphasias (16\%), with relatively few phonemic, mixed phonemic/ semantic, and neologistic errors (3-4\% each). Eleven other patients showed only mild anomia (patients P1-P11), on average failing to name $3 \%$ of the objects (ranging from 0 to $9 \%$ in individual patients). Another patient (P20) was excluded from both subgroups for reasons described below.

To further dissect the mechanisms of anomia, we sorted trials in each of the eight severely anomic patients (P12-P19) according to whether or not the target word referred to an object that was later named on confrontation (i.e., sorted by target rather than by cue). This analysis was not feasible in the mild anomia subgroup because there were too few unnamed objects. There was a trend toward lower accuracy in match/nonmatch judgments for unnamed than for named items ( $89 \%$ vs $95 \%$, respectively, $t_{(7)}=$ 2.1, $p=0.07$ ). Reaction times (based on trials with accurate responses) were significantly slower for unnamed items than for named items ( $1108 \mathrm{~ms}$ vs $\left.1005 \mathrm{~ms}, t_{(7)}=2.7, p=0.03\right)$. This slowing was mainly driven by slower responses to matching unnamed versus named items (1113 ms vs $915 \mathrm{~ms}, t_{(7)}=3.4, p=$ 0.01 ), whereas reaction times did not differ between named and unnamed items on trials with related mismatches $\left(t_{(7)}=1.3, p=\right.$ $0.24)$ or unrelated mismatches $\left(t_{(7)}=0.91, p=0.39\right)$.

ERPs to named and unnamed items computed for the severely anomic subgroup are shown in Figure 4, $a$ and $b$, calculated only for trials with correct responses during the recognition of the target word as a match or mismatch. Contrasts of matching versus mismatch amplitudes (350-550 ms, bilateral central/posterior cluster) revealed a significant $\mathrm{N} 400 \mathrm{~m}$ for named $\left(F_{(1,7)}=\right.$ $13.84, p=0.007)$ but not for unnamed $\left(F_{(1,7)}=1.31, p=0.29\right)$ items. The $\mathrm{N} 400 \mathrm{c}$ was unreliable for both named and unnamed items $\left(F_{(1,7)}=1.18, p=0.31 ; F_{(1,7)}=1.42, p=0.27\right)$.
Table 2. Correlations between $\mathrm{N} 400 \mathrm{~m}$ and language function

\begin{tabular}{lcc}
\hline & Correlation & $p$ value \\
\hline Naming and semantics & & \\
Boston Naming Test & 0.50 & $0.02^{*}$ \\
WAB Auditory Word Recognition & 0.57 & $0.009^{*}$ \\
Peabody Picture Vocabulary Test & 0.53 & $0.03^{*}$ \\
Pyramids \& Palm Trees -Pictures & 0.47 & $0.04^{*}$ \\
Pyramids \& Palm Trees -Verbal & 0.56 & $0.05^{*}$ \\
Production and syntax & & \\
WAB Spontaneous Speech & 0.28 & 0.25 \\
NAVS Sentence Production & -0.27 & 0.30 \\
NAVS Sentence Comprehension & -0.27 & 0.28 \\
Words per minute & -0.13 & 0.63 \\
Mean length of utterance & -0.19 & 0.44 \\
\hline${ }^{*} p<0.05$, two-tailed. & &
\end{tabular}

\section{b Severely anomic subgroup,} unnamed items
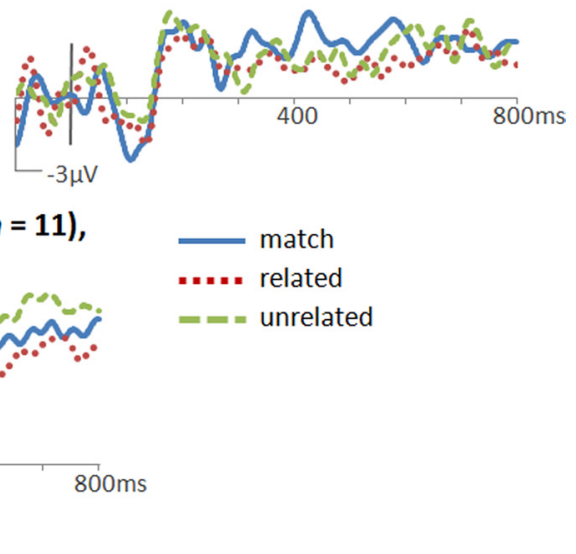

$800 \mathrm{~ms}$
Responses to successfully named items in severely anomic patients, mildly anomic patients, and controls

Were behavioral and N400 differences between the PPA and control groups driven by abnormalities in the severely anomic subgroup? In other words, were the mildly anomic patients more like severely anomic patients or more like controls? We examined these questions by comparing behavior and ERPs for accurately matched and correctly named items across subgroups (in contradistinction to previous group analyses, which included trials with unnamed items). Matching accuracy did not distinguish the mildly anomic and severely anomic subgroups (95\% vs $94 \%$, $\left.t_{(17)}=0.44, p=0.67\right)$, nor did it distinguish the mildly anomic subgroup and controls $\left(95 \%\right.$ vs $98 \%, t_{(11)}=1.7, p=$ 0.12 ). Reaction times were faster in the mildly anomic subgroup than in the severely anomic subgroup (798 ms vs 1005 $\left.\mathrm{ms}, t_{(15.3)}=2.3, p=0.04\right)$, but did not differ between the mildly anomic subgroup and controls (798 ms vs $686 \mathrm{~ms}, t_{(20.3)}=1.5, p=$ $0.14)$. Thus, slowing of reaction times in PPA, as found in the previous group comparison, was apparently driven by the severely anomic patients.

Mildly anomic patients showed a significant N400m (Fig. 4c) (match/mismatch contrast, named objects only, 350-550 ms, bilateral central/posterior cluster, $F_{(1,10)}=19.73, p=$ $0.001)$. The magnitude of this effect was not significantly different from that in the severely anomic subgroup $(2.9 \mu \mathrm{V}$ vs 
Table 3. Different patterns of successful and impaired object naming

\begin{tabular}{lclll}
\hline \multirow{2}{*}{ Group } & Reaction times (ms): & & & N400m \\
& Match/Rel/Unrel & N400c & N400m & timing \\
\hline Control & $630 / 722 / 705$ & + & + & Normal \\
PPA: recognized \& named (P11-P19) & $915 / 1087 / 1016$ & - & + & Prolonged \\
PPA: recognized \& unnamed (P11-P19) & $1113 / 1175 / 1058$ & - & - & Not present \\
PPA: recognized \& unnamed & $543 / 676 / 647$ & - & + & Normal \\
$\quad$ (Postsemantic, P20) & & & & \\
\hline
\end{tabular}

Patterns are classified according to three parameters: whether the target word is accurately recognized as a match or mismatch for the pictured object, whether it can be produced during naming aloud, and whether a name that cannot be produced during naming aloud can be produced in written form (postsemantic, as in the case of P20). Two of the patterns in this table are derived from a subset of patients, P11-P19, for reasons described in the text. Another pattern, not shown in this table, is one where anomia occurs on a background of severe word comprehension deficits where recognition of the word as a match or mismatch is also impaired. Patients with significant numbers of such recognition errors were not included in this study since they were unable to understand task instructions. This table illustrates the multiple patterns of impairment that can contribute to the object naming impairments of PPA.

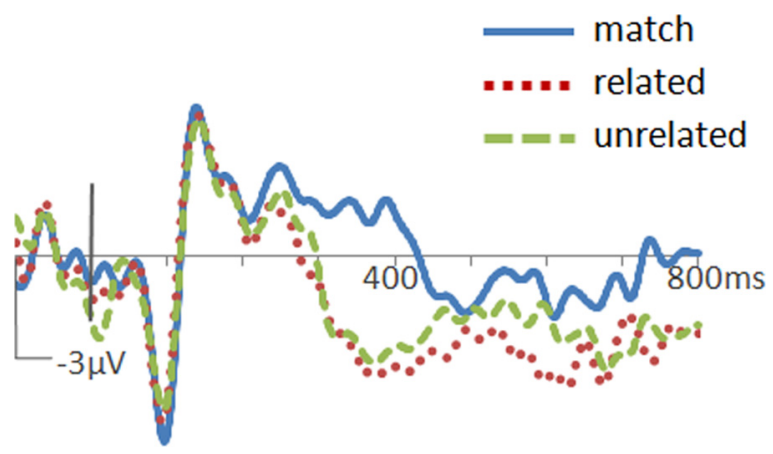

Figure 5. ERPs from a patient with postsemantic anomia at $C z$. This patient was unable to name $99 \%$ of the objects used during testing, but still showed an intact N400m to those items.

$2.4 \mu \mathrm{V}$ at $\left.\mathrm{Cz}, t_{(16)}=0.57, p=0.58\right)$ or in controls $(2.9 \mu \mathrm{V}$ vs $\left.3.3 \mu \mathrm{V}, t_{(21.2)}=0.45, p=0.66\right)$.

The timing and duration of N400m was assessed in $100 \mathrm{~ms}$ intervals for each PPA subgroup. Whereas N400m was found in controls from 300 to $500 \mathrm{~ms}$ (Fig. 3a), in mildly anomic patients it was delayed, reliable only at $400-500 \mathrm{~ms}\left(F_{(1,10)}=29.23, p<\right.$ $0.001)$. In severely anomic patients, it was delayed and lengthened, reliable at $400-500 \mathrm{~ms}\left(F_{(1,7)}=15.91, p=0.005\right), 500-600$ $\mathrm{ms}\left(F_{(1,7)}=18.72, p=0.003\right)$, and $600-700 \mathrm{~ms}\left(F_{(1,7)}=8.85, p=\right.$ 0.02 ). As in severely anomic patients, N400c was not apparent in mildly anomic patients (related/unrelated, $350-550 \mathrm{~ms}, t_{(10)}=$ $0.91, p=0.38$ ). In short, ERPs from mildly anomic patients showed the two key ERP results that distinguished PPA patients from controls in the whole-group analysis: N400m effects were delayed and N400c effects were absent.

\section{A case of postsemantic anomia}

One PPA patient demonstrated a neuropsychological profile that differed from that of all other PPA patients. This patient, P20, was unable to name $99 \%$ of the experimental objects during posttesting (Table 3) and showed the highest level of anomia in the present PPA sample. She was also only able to name 16 of 60 items on the BNT. However, when we allowed her to write the names rather than saying them aloud, she was able to write 49 of 60 items. This suggested that her anomia may stem from postsemantic impairments in word production. Consistent with this, patient P20 performed with near-ceiling accuracy on semantic-level neuropsychological tests such as the WAB Auditory Word Recognition, and on the verbal and object versions of the PPT (Table 1). ERPs from this patient are shown in Figure 5. Unlike the results with unnamed items in the severely anomic subgroup, patient P20 showed a clear N400m for items that she was unable to name aloud, consistent with a profile of postsemantic rather than associative impairments in naming.

\section{Discussion}

Primary progressive aphasia is a language-based dementia characterized by the gradual and initially selective degeneration of the left perisylvian language network (Mesulam, 2003). Object naming is one of the most distributed functions of the language network and also one of the most frequent areas of impairment in PPA. In this electrophysiological study of PPA, we investigated object naming with a task that required participants to decide whether a noun matched the preceding picture of an object. Participants were not required to produce the name of the object at the time of ERP recordings, but only to judge whether the presented name was the correct one or not (thus avoiding EEG speech artifacts). The semantic relationship on each trial was systematically varied-in some trials the word was the name of the object (match condition), in others it was a mismatch that belonged to the same semantic category (related condition), and in still others it was both a mismatch and a member of a different category (unrelated condition). Our hypotheses focused on exploring whether electrophysiological indices of word-object priming in PPA were related to more traditional measures of word comprehension, whether they differed in response to named versus unnamed objects, and whether their preservation was necessary to support object naming.

Task performance at the matching stage was highly accurate in PPA patients, allowing for analysis of ERPs and reaction times for correct responses only (eliminating a potential confound that often impacts patient studies). Responses in both groups were faster for matching than for mismatched words, and also faster for unrelated than for related mismatches, indicating that participants in both groups perceived semantic relationships between words and objects.

The N400 amplitudes were sensitive to the semantic distance between associations evoked by the objects and those evoked by the words. An electrophysiological N400m effect, reflecting differential responses as a function of whether or not the word matched, was found in both groups. The face validity of the $\mathrm{N} 400 \mathrm{~m}$ amplitude as an index of semantic processing was confirmed by showing that it correlated with performance on tests of object and word knowledge (WAB word recognition, PPT, and PPVT), but not with postsemantic aspects of naming such as fluency and grammar.

High accuracy in the picture-word matching task indicated that patients in this sample were able to recognize the word that matched the object. PPA patients with more severe semantic impairments tend to fail such recognition tasks (Mesulam et al., 2009). Patients with severe single word comprehension deficits were not included in the present sample because they were unable to follow task instructions. Nonetheless, the PPA patients who were included displayed a wide spectrum of naming ability ranging from 5 to $98 \%$ accuracy in a standard test of naming (the BNT). To determine whether the patients could not only recognize the name of the object during the ERP recording phase but also produce it during confrontation naming, patients were asked to name the experimental pictures aloud following the ERP recording session. Eight patients (P12-P19) stood out from the rest because of the high number of objects they were unable to name aloud during the post-ERP testing. In the severely anomic subgroup, N400m was evident, but only for trials involving objects that could be named. The $\mathrm{N} 400 \mathrm{~m}$ was not triggered by words that could not be produced during confrontation naming even 
though the same words had been recognized correctly as the name of the object during the matching phase. Differential ERP responses to matching versus mismatched words thus appeared to reflect processing that was tightly tied to the ability to access rather than recognize (or understand) an object's name. Reaction times were also faster for trials involving objects that could be named than for those that could not be named. For the latter type of trial, object knowledge was apparently sufficient for accurate matching performance but insufficient for naming or for the semantic processing indexed by $\mathrm{N} 400 \mathrm{~m}$. Thus, the association of an object to its name reflects a multiplicity of cognitive processes.

The question may arise whether N400m can be exhibited with objects that cannot be named exclusively on the basis of postsemantic factors such as phonological encoding or articulatory programming. This question was addressed in a serendipitously encountered individual with PPA, patient P20, who was only able to name $27 \%$ of all items on the BNT by confrontation, but could write the names of $82 \%$ of all items. This dissociation indicated that her confrontation naming failures reflected "postsemantic" deficits. Unlike the general pattern seen with words that could not be named by patients with severe anomia, patient P20 showed a clear N400m to items that she was unable to name. Accordingly, we conclude that naming failures in the presence of intact $N 400 \mathrm{~m}$ effect strongly implicate postsemantic impairments at a stage subsequent to lexical access.

Conversely, correct confrontation naming does not imply that semantic processing is normal. The $\mathrm{N} 400 \mathrm{~m}$ was prolonged in the PPA patients, even for items that were correctly named. In addition, controls but not PPA patients showed an N400c effect: more negative responses to unrelated than to related mismatch words. N400c was absent in PPA, even for trials with objects that could be named in both the mildly and severely anomic subgroups. $\mathrm{N} 400 \mathrm{c}$ is of lower magnitude than the $\mathrm{N} 400 \mathrm{~m}$ in neurologically intact controls, and is based on the ability to differentially encode whether or not mismatched words came from the same category as the object, so it is not surprising that this relatively subtle distinction of differential semantic distances is vulnerable to neuronal loss within the left-hemisphere language network.

Hagoort et al. (1996) found a differential response to related versus unrelated auditory word pairs in patients with aphasia due to cerebrovascular accident, a setting where a lesion is produced abruptly in a clearly delineated region and where compensatory changes become operative almost immediately. The situation is quite different in PPA, where the neuronal loss is progressive and where many components of the language network are simultaneously affected. There were also differences in the tasks: the patients of Hagoort et al. (1996) passively listened to word pairs, whereas PPA patients in the present study judged object-word pairs. Further studies directly comparing N400 effects in word pairs versus object-word pairs would be informative.

It is interesting that N400c abnormalities were observed even for objects that PPA patients could successfully name. This finding is consistent with previous work from our laboratory on PPA, which showed impaired priming and abnormal semantic interference even in patients who performed normally on conventional tasks of word comprehension (Vandenberghe et al., 2005; Rogalski et al., 2008). Although PPA patients in the current study failed to show an N400c, they were slower to respond on related than on unrelated trials, suggesting that category membership was being encoded but not in a manner that could be indexed by the N400.

Neurological diseases have offered unique opportunities for exploring the biological bases of cognitive function. Such inves- tigations were initially conducted on patients with focal cerebrovascular disease. Recently, neurodegenerative diseases have become the focus of equally fruitful investigations. Neurodegenerative diseases such as those that cause PPA tend to have selective predilections for specific neuronal types, cortical layers, and even neural systems. Moreover, the primary damage is almost always confined to gray matter and does not spread to white matter, as frequently happens in cerebrovascular accidents. No area undergoing neurodegeneration sustains a complete cessation of activity. Instead, the progressive neuronal loss leads to a gradual dissolution of function at the same time that the affected neural circuits undergo some degree of compensatory reorganization (Sonty et al., 2003; Vandenbulcke et al., 2005). Investigations of these patients are uniquely interesting for exploring the cognitive architecture of naming, especially since the partial perturbations induce subtle dissociations that are less frequently seen in patients with cerebrovascular disease.

Overt naming cannot proceed without the hypothetical semantic linkage of an object percept to its corresponding lexical representation. The nearly perfect object-word matching scores of our patients indicated that these linkages were being established accurately at the behavioral level even by the most anomic patients in the group. However, the electrophysiological signals exhibited during this process revealed additional qualitative differences. Even the least anomic patients, for example, showed abnormalities in the neuronal substrates of linkage with word meaning, as characterized by delayed $\mathrm{N} 400 \mathrm{~m}$ and negligible N400c. Severely anomic patients displayed more profound abnormalities as revealed by the further temporal smearing of the $\mathrm{N} 400 \mathrm{~m}$, reflecting delayed neuronal responses or a decrease in the sharpness of the semantic tuning curve. Neuronal correlates of semantic linkages were further compromised for objects that could not be named overtly in the severely anomic group, evidenced by a nonsignificant N400m to those items. In contrast, N400m was intact for items that could not be named because of exclusively postsemantic impairments, as in the case of one patient who could write object names she could not produce verbally. These results, obtained in a unique group of patients with a progressive degeneration of the language network, reveal novel details of the neurophysiological architecture of associative linkages that mediate confrontation naming in health and disease.

\section{References}

Auchterlonie S, Phillips NA, Chertkow H (2002) Behavioral and electrical brain measures of semantic priming in patients with Alzheimer's disease: implications for access failure versus deterioration hypotheses. Brain Cogn 48:264-267.

Damasio H, Tranel D, Grabowski T, Adolphs R, Damasio A (2004) Neural systems behind word and concept retrieval. Cognition 92:179-229.

DeLeon J, Gottesman RF, Kleinman JT, Newhart M, Davis C, Heidler-Gary J, Lee A, Hillis AE (2007) Neural regions essential for distinct cognitive processes underlying picture naming. Brain 130:1408-1422.

Dunn LA, Dunn LM (2006) Peabody Picture Vocabulary Test-4. Circle Pins, MN: American Guidance Service.

Ford JM, Askari N, Mathalon DH, Menon V, Gabrieli JDE, Tinklenberg JR, Yesavage J (2001) Event-related brain potential evidence of spared knowledge in Alzheimer's disease. Psychol Aging 16:161-176.

Grossman M, McMillan C, Moore P, Ding L, Glosser G, Work M, Gee J (2004) What's in a name: voxel-based morphometric analyses of MRI and naming difficulty in Alzheimer's disease, frontotemporal dementia and corticobasal degeneration. Brain 127:628-649.

Hagoort P, Brown CM, Swaab TY (1996) Lexical-semantic event-related potential effects in patients with left hemisphere lesions and aphasia and patients with right hemisphere lesions without aphasia. Brain 119: $627-649$. 
Howard D, Patterson K (1992) Pyramids and Palm Trees: a test of semantic access from pictures and words. Bury St. Edmonds, Suffolk, UK: Thames Valley Test Company.

Kaplan E, Goodglass H, Weintraub S (1983) The Boston Naming Test. Philadelphia: Lea and Febiger.

Kertesz A (1982) Western Aphasia Battery. San Antonio, TX: The Psychological Corporation.

Kiang M, Kutas M, Light GA, Braff DL (2008) An event-related brain potential study of direct and indirect semantic priming in schizophrenia. Am J Psychiatry 165:74-81.

Kutas M, Hillyard SA (1980) Reading senseless sentences: brian potentials reflect semantic incongruity. Science 207:203-205.

Levelt WJM, Roelofs A, Meyer AS (1999) A theory of lexical access in speech production. Behav Brain Sci 22:1-75.

Mathalon DH, Faustman WO, Ford JM (2002) N400 and automatic semantic processing abnormalities in patients with schizophrenia. Arch Gen Psychiatry 59:641-648.

McMillan C, Gee J, Moore P, Dennis K, DeVita C, Grossman M (2004) Confrontation naming and morphometric analyses of structural MRI in frontotemporal dementia. Dement Geriatr Cogn Disord 17:320-323.
Mesulam MM (1982) Slowly progressive aphasia without generalized dementia. Ann Neurol 11:592-598.

Mesulam MM (2003) Primary progressive aphasia—a language-based dementia. N Engl J Med 349:1535-1542.

Mesulam MM, Rogalski E, Wieneke C, Cobia D, Rademaker A, Thompson C, Weintraub S (2009) Neurology of anomia in the semantic variant of primary progressive aphasia. Brain 132:2553-2565.

Rogalski E, Rademaker A, Mesulam MM, Weintraub S (2008) Covert processing of words and pictures in nonsemantic variants of primary progressive aphasia. Alzheimer Dis Assoc Disord 22:343-351.

Sonty SP, Mesulam MM, Thompson CK, Johnson NA, Weintraub S, Parrish TB, Gitelman DR (2003) Primary progressive aphasia: PPA and the language network. Ann Neurol 53:35-49.

Vandenberghe RR, Vandenbulcke M, Weintraub S, Johnson N, Porke K, Thompson CK, Mesulam MM (2005) Paradoxical features of word finding difficulty in primary progressive aphasia. Ann Neurol 57:204-209.

Vandenbulcke M, Peeters R, Van Hecke P, Vandenberghe R (2005) Anterior temporal laterality in primary progressive aphasia shifts to the right. Ann Neurol 58:362-370. 